\title{
Symmetry incorporated Fuzzy C-means Method for Image Segmentation
}

\author{
Surani Anuradha Jayasuriya and Alan Wee-Chung Liew \\ School of Information and Communication Technology, Griffith University, Gold Coast Campus \\ QLD 4222, Australia
}

\begin{abstract}
This paper presents a new modified fuzzy c-means (FCM) clustering algorithm that exploits bilateral symmetry information in image data. With the assumption of pixels that are located symmetrically tend to have the same intensity values; we compute the degree of symmetry for each pixel with respect to a global symmetry axis of the image. This information is integrated into the objective function of the standard FCM algorithm. Experimental results show the effectiveness of the approach. The method was further improved using neighbourhood information, and was compared with conventional fuzzy c-means algorithms.
\end{abstract}

Keywords - Fuzzy C-means; bilateral symmetry; image segmentation

\section{INTRODUCTION}

Image segmentation is an important first step in many image analysis applications such as object recognition, pattern detection and medical imaging. These applications demand for accurate, robust and efficient algorithms. However, it is still a challenging task due to various noise artifacts and complexity of images.

Fuzzy C-means algorithm [1] is widely used as a soft segmentation method due to its efficiency and ability to handle ambiguity by incorporating fuzziness for the belongingness of each image pixel. In this way, FCM can retain more information from the original image in comparison with crisp or hard segmentation methods. However, one disadvantage of standard FCM is that it uses only image intensity values without incorporating any spatial information in image context that makes it sensitive to noise. In order to overcome this drawback, a number of other methods [2-4], have been proposed by integrating spatial information in to the standard FCM objective function. Most of these are based on the consideration of immediate neighborhood information of pixels. Although these methods have been proven to be effective, they still lack enough robustness to noise due to the unavailability of higher level geometric information in the image.

Symmetry is well known as a powerful mechanism that facilitates identifying the structure of an object [5]. It is an intrinsic property of an object that remains invariant to certain classes of transformations. Although a number of work has exploited symmetry of the image for certain recognition tasks [6-7], symmetry integrated generalized image segmentation field is still immature [8]. A recent work [8] based on regiongrowing technique integrates bilateral symmetry in to the image segmentation algorithm. In their work, symmetry axis is detected using the global symmetric constellations of features [9]. With respect to this symmetry axis, a symmetry affinity matrix is created which is then integrated into a homogeneity criterion in the region growing algorithm along with colour and texture.

In this paper, by incorporating bilateral symmetry information in image context, we propose a modified FCM algorithm for image segmentation. We assume that a global bilateral symmetry axis of the image is detected and its location is known. For normal images, there are a number of methods proposed for this task [9-10]. To identify the accurate location of the symmetry plane in brain images, we use the method described in our previous paper [11-12]. In standard FCM algorithm, the classification of each pixel is determined by its intensity value. In our proposed method, the classification of each pixel is also influenced by the pixel in a symmetrical position with respect to the global symmetry axis. We call this pixel a mirror pixel. This is realized by measuring a degree of symmetry for each pixel. The aim of this paper is to show the feasibility and robustness of the algorithm with the addition of symmetry information. In order to show the improvement of the method, we then further extended the algorithm by considering neighborhood spatial information. In experiments, some synthetic and simulated brain images and real images were used to assess the performance of the new method in comparison with conventional FCM algorithms.

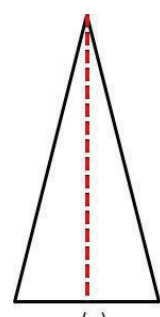

(a)

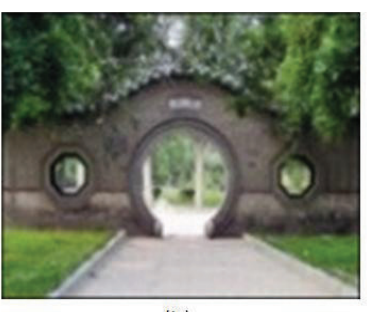

(b)

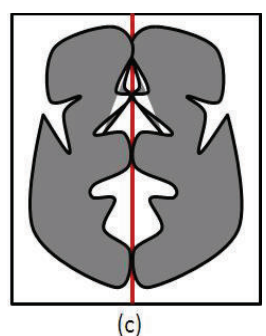

(c)
Fig. 1. Examples of images with bilateral symmetry. (b) is adapted from [8] 


\section{METHODOLOGY}

\section{A. Standard Fuzzy C-means Algorithm (FCM)}

The FCM algorithm [9, 10] assigns pixels to each category by using fuzzy memberships. Let $X=\left\{x_{k}, k=1,2, . . N\right\}$ denotes an image with $N$ pixels to be partitioned into $c$ clusters. $X_{k}$ is the intensity value of pixel $k$. The FCM algorithm is an iterative optimization that classifies $X$ into $c$ fuzzy clusters by minimizing the following objective function with respect to the membership function $\boldsymbol{u}$ and centroid values $\boldsymbol{v}$

$J_{F C M}=\sum_{i=1}^{c} \sum_{k=1}^{N} u_{i k}^{m}\left\|x_{k}-v_{i}\right\|^{2}$

$u_{i k}$ is the fuzzy membership value of pixel $k$ in cluster $i$, satisfying $0 \leq u_{i k} \leq 1$, and $\sum_{i=1}^{c} u_{i k}=1 . v_{k}, k=1, . . c$ denotes the average intensity value in cluster $k .\|$.$\| is a norm metric, and$ we have considered Euclidean distance here. The parameter $m$ is a weighting exponent on each fuzzy membership and determines the amount of "fuzziness" of the resulting classification. Generally, $m=2$, and it is used in this study.

This objective function is minimized when pixels close to the centroid of their clusters are assigned high membership values while pixels far from the centroid are assigned low membership values. The update equations for minimizing $J_{F C M}$ with the necessary conditions are as follow.

$$
u_{i k}=\frac{1}{\sum_{i=1}^{c}\left(\frac{\left\|x_{k}-v_{i}\right\|}{\left\|x_{k}-v_{i}\right\|}\right)^{2 / m-1}}
$$

$$
v_{i}=\frac{\sum_{k=1}^{N} u_{i k}^{m} x_{k}}{\sum_{k=1}^{N} u_{i k}^{m}}
$$

\section{B. Modified FCM with Symmetry Information (symFCM)}

The integration of information from the image context is important for accurate image segmentation. When segmenting symmetrical or nearly symmetrical image objects, this symmetry information can be utilized to give better segmentation in noisy images.

With our algorithm, we assume that the location of the global symmetry plane of the symmetrical/approximate symmetrical image is known. Then, for each pixel a degree of symmetry value is computed. Although various symmetry measures can be used here [10], we defined a symmetry measure as follow

$\beta_{k}=1-\gamma_{k}$ where

$$
\begin{aligned}
& \begin{aligned}
\gamma_{k} & =\frac{1}{N_{R}+1}\left(\left\|x_{k}-x_{k^{\prime}}\right\|+\sum_{r \in N_{k}}\left\|x_{r}-x_{r^{\prime}}\right\|\right) \\
& =\left\|\bar{x}_{k}-\bar{x}_{k^{\prime}}\right\|
\end{aligned} \\
& \text { Here } \bar{x}_{t}=\frac{1}{N_{R}+1}\left(x_{t}+\sum_{r \in N_{t}} x_{r}\right)
\end{aligned}
$$

$\beta_{k}$ is the degree of symmetry for $k$ th pixel, and $k^{\prime}$ is the mirror pixel of $k$ (see Fig. 2) . $N_{k}$ stands for the set of neighbours in the considering window and $N_{R}$ is the cardinality of $N_{k}$.

We propose a modification to FCM by introducing an additional term that allows the labeling of a pixel to be influenced by the label of its mirror pixel. We define a weighting function as follow.

$\alpha(\beta)=\frac{1}{1+e^{-(\beta-\mu) / \sigma}}$

$\mu$ is the global averaged symmetry value of the image. $\sigma$ controls the influence of the mirror pixel, and here we have used the standard deviation of the degree of symmetry values. By setting a threshold value to $\mu$, the symmetry influence can completely be turned off, if $\mu<$ threshold.

In a certain pixel, if the degree of symmetry $\beta$ is large, then $\alpha \rightarrow 1$ whereas for small $\beta, \alpha \rightarrow 0$. Since the weighting function can be pre-computed, the computation time of the algorithm can greatly be reduced.

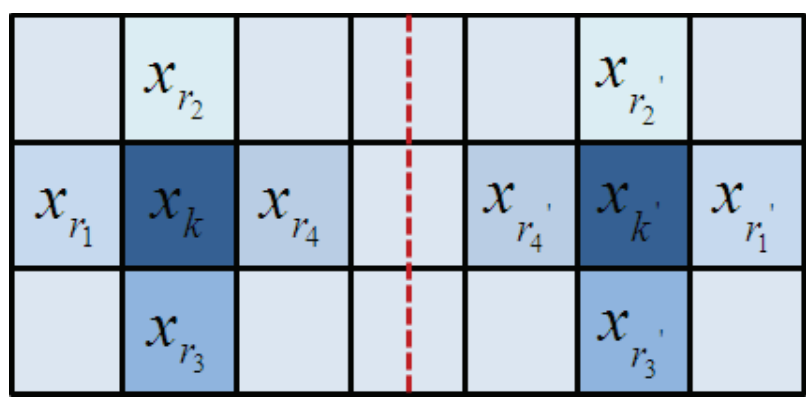

Fig. 2. Degree of symmetry is measured considering a neighnorhood of each pixel: an example with a first-roder neighborhood.

Based on this symmetry information, our modified FCM (SymFCM) objective function can be defined as follow.

$$
J_{\text {symFCM }}=\sum_{i=1}^{c} \sum_{k=1}^{N} u_{i k}^{m}\left\|x_{k}-v_{i}\right\|^{2}+\alpha_{k} u_{i k}^{m}\left\|x_{k^{\prime}}-v_{i}\right\|^{2}
$$


Thus, the update equations for minimizing $J_{\text {SymFCM }}$ with the necessary conditions are as follow.

$$
\begin{gathered}
u_{i k}=\frac{1}{\sum_{i=1}^{c}\left(\frac{\left(\left\|x_{k}-v_{i}\right\|^{2}+\alpha_{k}\left\|x_{j}-v_{i}\right\|^{2}\right)}{\left(\left\|x_{k}-v_{i}\right\|^{2}+\alpha_{k}\left\|x_{j}-v_{i}\right\|^{2}\right)}\right)^{1 / m-1}} \\
v_{i}=\frac{\sum_{k=1}^{N} u_{i k}^{m}\left(x_{k}+\alpha_{k} x_{j}\right)}{\sum_{k=1}^{N} u_{i k}^{m}\left(1+\alpha_{k}\right)}
\end{gathered}
$$

This modified symFCM for image segmentation can be summarized in the following steps.

Step1. Read the brain volume data.

Step2. Set the number of tissue classes $c$, select initial cluster centroids, and set the $\varepsilon$ to a small value $\left(1 \times 10^{-5}\right)$.

Step3. Compute the degree of symmetry matrix.

Step4. Update fuzzy membership $u_{i k}$ using eq.(7)

Step5. Update cluster centroids $v_{i}$ using eq.(8).

Step6. If $\left\|u_{i k}^{\text {new }}-u_{i k}^{\text {old }}\right\| \leq \varepsilon$, proceed to step 7, else return to step4.

Step7. Get the final segmentation results using the maximum fuzzy membership value of each pixel.

\section{Extended symFCM with Neighboring Information (S_symFCM)}

Since the pixels on an image is highly correlated neighboring spatial information becomes important in image segmentation. Therefore, in order to improve our symmetry integrated method further, we incorporated neighborhood information as an additional term to the symFCM objective function.

We adapted the spatial continuity incorporated clustering method described in [13]. This technique is based on an adaptive dissimilarity index that takes into account the influence of the neighboring pixels in a $3 \times 3$ window. Its adaptive index is capable of enhancing the influence of the neighboring pixels on the center pixel depending on the neighborhood window is either in a homogeneous region, or not.

Incorporating this spatial continuity condition into our symFCM, we define the new objective function S_symFCM as follow.

$$
\begin{array}{r}
J_{s_{-} \text {symFCM }}=\sum_{i=1}^{c} \sum_{k=1}^{N} u_{i k}^{m}\left\|x_{k}-v_{i}\right\|^{2}+\alpha_{k} u_{i k}^{m}\left\|x_{j}-v_{i}\right\|^{2}+ \\
\lambda u_{i k}^{m}\left\|x_{r m}-v_{i}\right\|
\end{array}
$$

Where $x_{r m}=\frac{1}{N_{R}} \sum_{r \in N_{k}} x_{r}$.

Here, for computational efficiency, we have used the mean value of the neighbors $\left(x_{r m}\right)$ by assuming that the number of edges are small in comparison with the smoothed region.

$\lambda$ is the dissimilarity index explained in [13], and here we have adapted the parameters as follow.

$\lambda=\frac{1}{1+e^{\left(\partial-\mu_{s}\right) / \sigma_{s}}}$

Here,

$\partial(k)=\left(\frac{1}{N_{R}} \sum_{r \in N_{k}}\left\|x_{k}-x_{r}\right\|^{2}\right) \quad r \in N_{k}$, and

$\mu_{s}=\frac{1}{N} \sum_{k=1}^{N} \partial(k)$.

For the steepness parameter $\sigma_{s k}$, we used the standard deviation of the distances $\left\|x_{k}-x_{r}\right\|^{2}$ in the neighboring window.

Then, the update equations for $J_{s_{-} \text {symFCM }}$ can be derived as follow.

$$
u_{i k}=\frac{1}{\sum_{i=1}^{c}\left(\frac{\left(\left\|x_{k}-v_{i}\right\|^{2}+\alpha_{k}\left\|x_{j}-v_{i}\right\|^{2}+\lambda\left\|\bar{x}_{k}-v_{i}\right\|^{2}\right)}{\left(\left\|x_{k}-v_{i}\right\|^{2}+\alpha_{k}\left\|x_{j}-v_{i}\right\|^{2}+\lambda\left\|\bar{x}_{k}-v_{i}\right\|^{2}\right)}\right)^{1 / m-1}}
$$

$v_{i}=\frac{\sum_{k=1}^{N} u_{i k}^{m}\left(x_{k}+\alpha_{k} x_{j}+\lambda \bar{x}_{k}\right)}{\sum_{k=1}^{N} u_{i k}^{m}\left(1+\alpha_{k}+\lambda\right)}$

The steps in the extended algorithm are similar to procedure in symFCM, except for computing a mean filtered image and the parameter $\lambda$ for neighborhood term. Membership values and centroids are updated using eq. (11) and (12) respectively.

\section{EXPERIMENTAL RESULTS}

In our experiments, we chose the parameters to be $\mathrm{m}=2$ and $\varepsilon=10^{-5}$. A $3 \times 3$ window of image pixels was considered for computation of degree of symmetry. In order to evaluate the validity of our approach, first we used $2 \mathrm{D}$ synthetic grayscale images which have either global symmetry axis or a global approximate symmetry axis. 
In order to measure the quality of the segmentation performance quantitatively, we also apply segmentation accuracy measure $(S A)$ given by,

$$
S A=\frac{\text { Number of correctly classified pixels }}{\text { Total number of pixels }} \times 100 \%
$$

Fig.3. illustrates the clustering results of a symmetrical synthetic image corrupted by Gaussian noise with variance 0.03. Standard FCM (Fig. 3(c)) is greatly affected by the noise $(S A=98.91 \%)$. In comparison, our method symFCM (Fig. 3(d)) is far more robust to noise $(S A=99.90 \%)$. Although the method SFCM with neighborhood information described in [13] removes most of the noise (Fig. 3(e)), the result is still not perfect $(S A=99.96 \%)$. Adopting this neighborhood information into our algorithm, the extended S_symFCM method gives almost perfect results $(S A=99.99 \%)$.

We also applied the same algorithms to a few other synthetic images which include non-symmetrical images. These results are illustrated in Fig.4-Fig.6.

In addition to this we also tested the algorithm on simulated brain images. The simulated MR images were obtained from the McConell Brain Imaging Center at the Montreal Neurological Institute, McGill University. (BrainWeb: http://www.bic.mni.mcgill.ca/brainweb/) [14]. The size of each image volume was $181 \times 217 \times 181$ (in mm) and slice thickness was $1 \mathrm{~mm}$. Various noise images with zero INU were taken for the experiment in order to analyze the effect of noise. Prior to segmentation, the extra-cranial tissues such as skull and meninges were removed from all images. Then, the brain images were supposed to be classified into three tissue classes: Gray Matter (GM), White Matter (WM), and Cerebrospinal Fluid (CSF). These results are shown in Fig.7 and Fig. 8.

Furthermore, we used several real images taken from the UCB database (The Berkeley segmentation dataset and benchmark) [15-16]. Fig. 9 and Fig.10 shows these results.

\section{A. Qualitative Evaluation}

According to the results shown in Fig.3-Fig.8, when a qualitative comparison is done, it can be seen that the proposed method shows better segmentation in comparison with the results obtained from standard FCM. Furthermore, extended method with neighboring information (S_symFCM) shows better results than the SFCM.

Even when non-symmetrical images are used, although there are some misclassified regions due to the consideration of symmetry, symFCM still achieves better overall segmentation comparing to FCM and S_symFCM shows further improved results.

\section{B. Quantitative Evaluation}

Table I depicts the segmentation accuracy $S A$ of the proposed methods (symFCM and S_symFCM), the standard FCM and SFCM when applied to synthetic images, and also brain simulated images. SA value is computed over 30 runs over each test, and taken the averaged segmentation accuracy value. The $S A$ columns show that as the percentage of noise is increased, the accuracy for all methods also decreases. symFCM, however, is much more robust to increased noise than the conventional FCM. S_symFCM performs better than the other methods under all noise conditions.

Table I. Segmentation accuracy $(S A)$ of each method

\begin{tabular}{|l|l|l|l|l|}
\hline Image & FCM & symFCM & S_FCM & S_symFCM \\
\hline Fig.3 & $98.91 \%$ & $99.90 \%$ & $99.96 \%$ & $99.99 \%$ \\
\hline Fig.4 & $98.91 \%$ & $99.81 \%$ & $99.98 \%$ & $99.98 \%$ \\
\hline Fig.5 & $88.96 \%$ & $95.71 \%$ & $97.63 \%$ & $98.98 \%$ \\
\hline Fig.6 & $88.04 \%$ & $94.91 \%$ & $97.32 \%$ & $98.02 \%$ \\
\hline Fig.7 & $91.33 \%$ & $92.09 \%$ & $93.78 \%$ & $93.93 \%$ \\
\hline Fig.8 & $86.67 \%$ & $88.18 \%$ & $89.89 \%$ & $90.42 \%$ \\
\hline
\end{tabular}

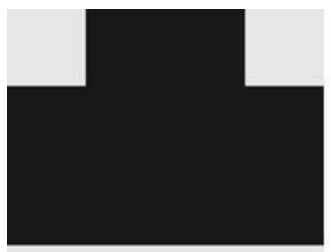

(a)
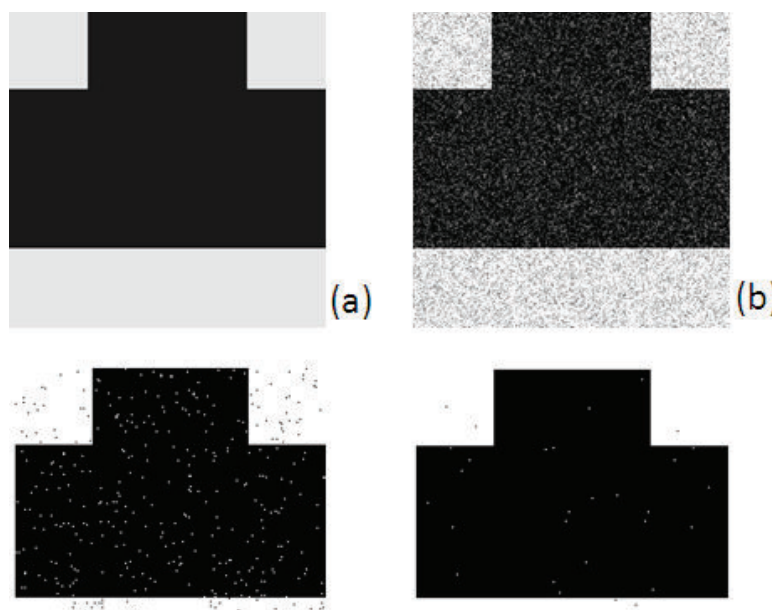

(c)

(d)
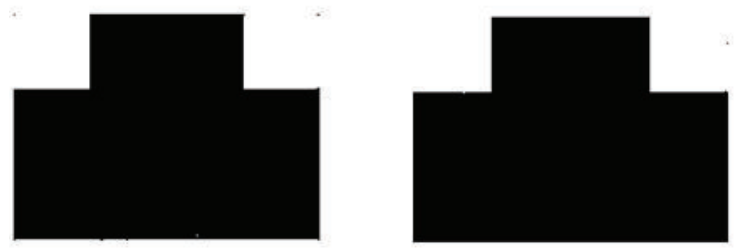

(e)

(f)

Fig. 3. Clustering results of a synthetic perfectly symmetrical image. (a). Original image, (b). the same image with gaussian noise (var=0.03), (c). FCM results, (d). symFCM results, (e). SFCM results, and (f). S_symFCM results. 


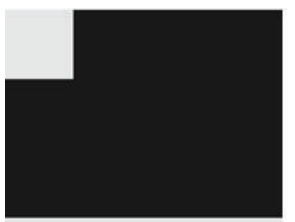

(a)
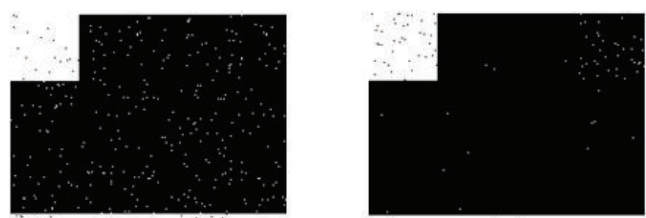

(c)

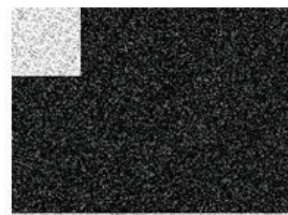

(b)

(d)
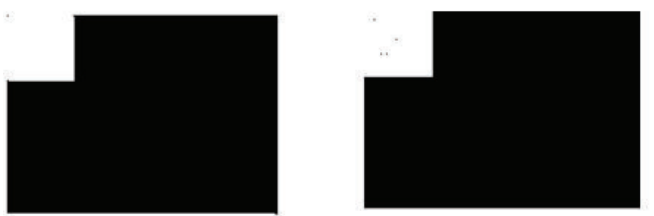

(e)

(f)

Fig. 4. Clustering results of a synthetic nearly symmetrical image. (a). Original image, (b). the same image with gaussian noise (var=0.03), (c). FCM results, (d). symFCM results, (e). SFCM results, and (f). S_symFCM results.
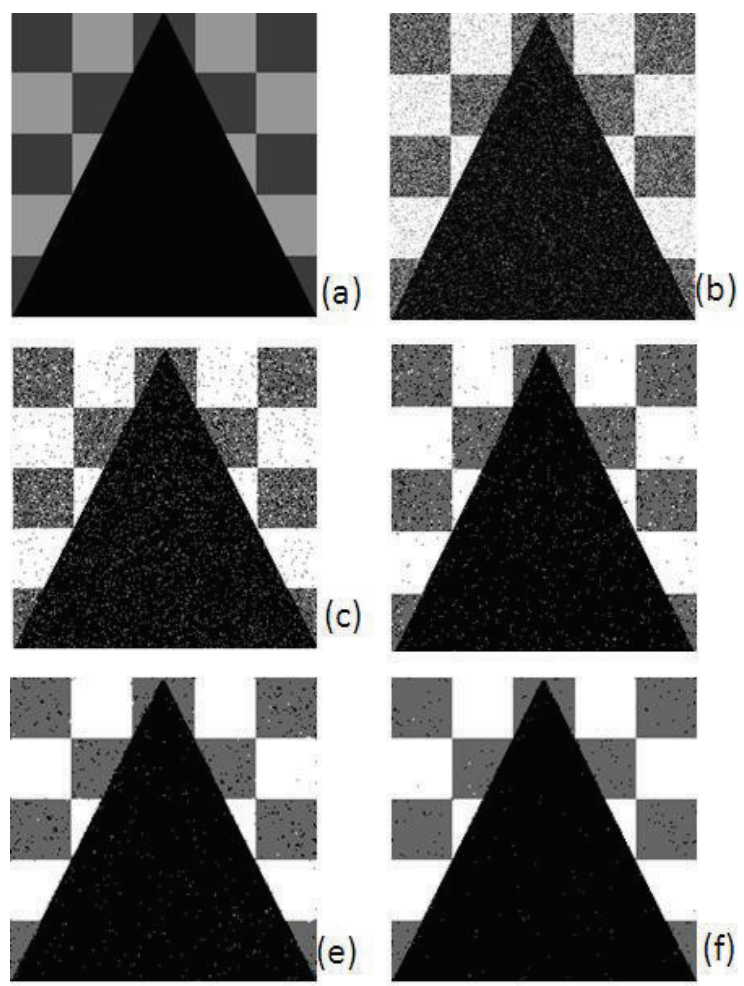

Fig. 5. Clustering results of a synthetic image. (a). Original image, (b). the same image with gaussian noise (var=0.03), (c). FCM results, (d). symFCM results, (e). SFCM results, and (f). S_symFCM results.
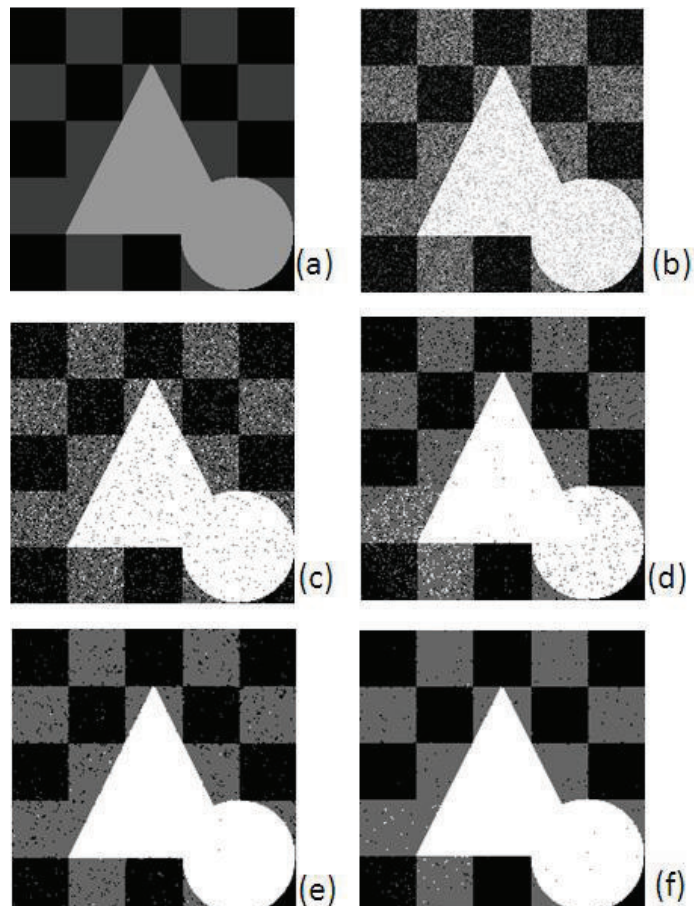

Fig. 6. Clustering results of a synthetic non-symmetrical image. (a). Original image, (b). the same image with gaussian noise (var=0.03), (c). FCM results, (d). symFCM results, (e). SFCM results, and (f). S_symFCM results.
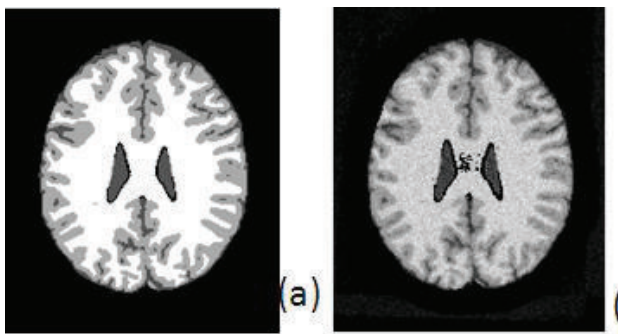

(b)
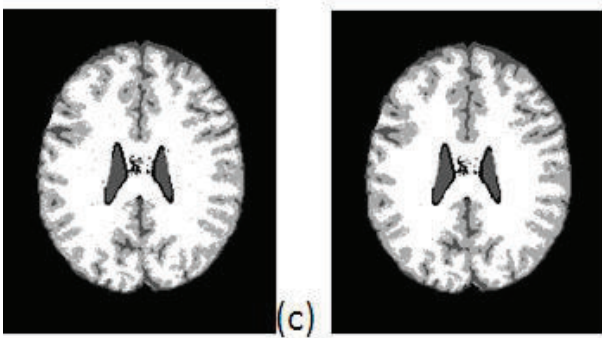

(d)
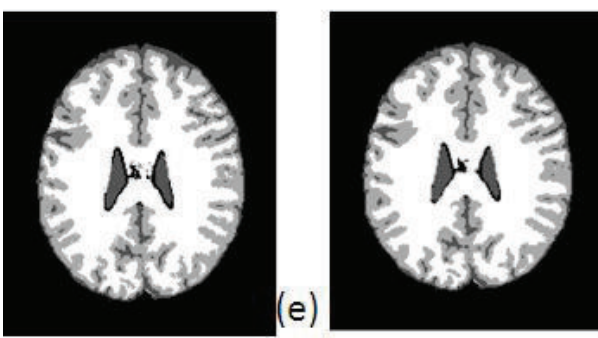

(f)

Fig. 7. Clustering results of a simulated brain MRI image. (a). Ground truth, (b). Simulated brain image with Noise 7\%, (c). FCM results, (d). symFCM results, (e). SFCM results, and (f). S_symFCM results. 


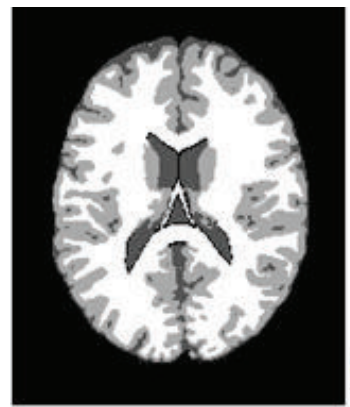

(a)

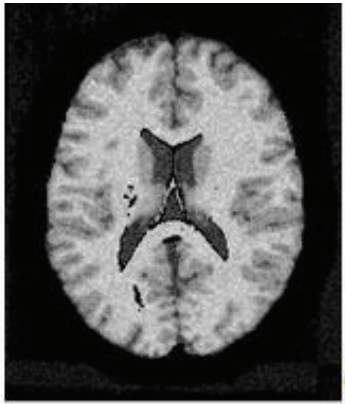

(b)

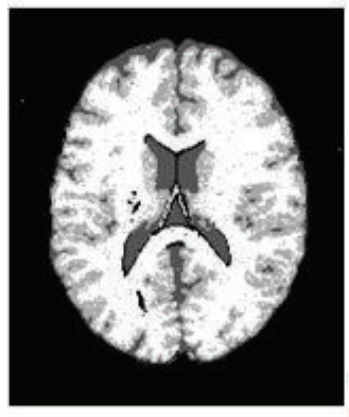

(c)
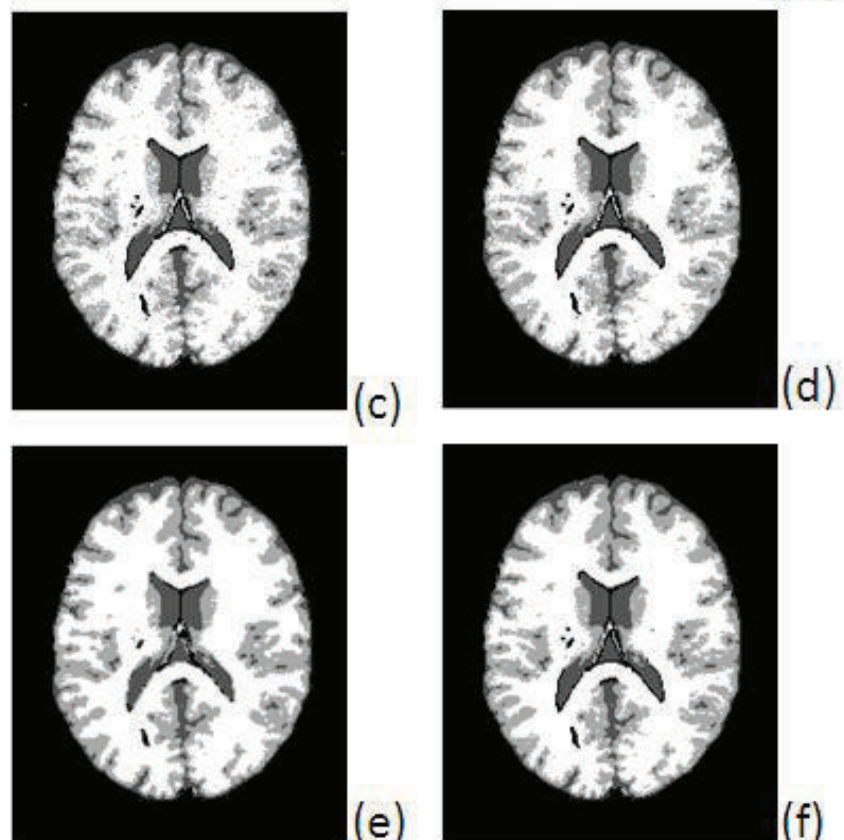

(e)

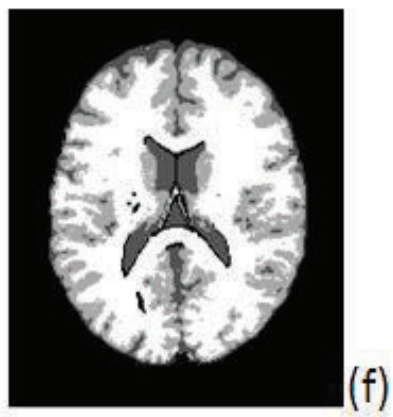

Fig. 8. Clustering results of a simulated brain MRI image. (a). Ground truth, (b). Simulated brain image with Noise $9 \%$, (c). FCM results, (d). symFCM results, (e). SFCM results, and (f). S_symFCM results.
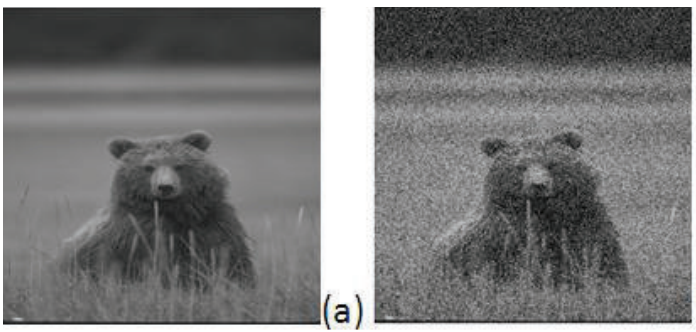

(b)
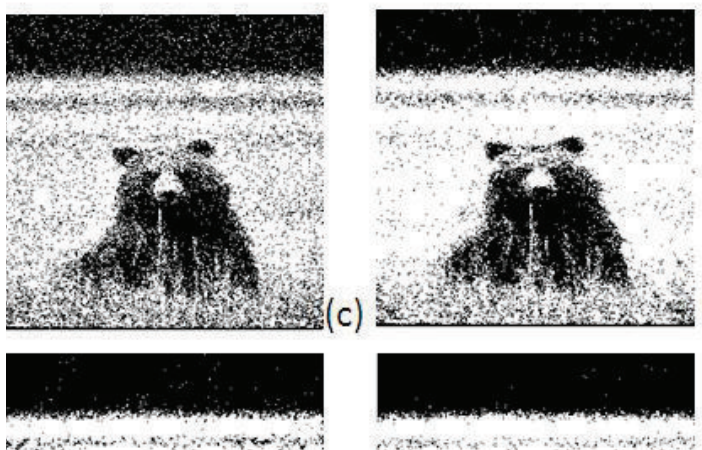

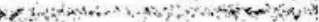

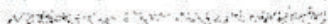
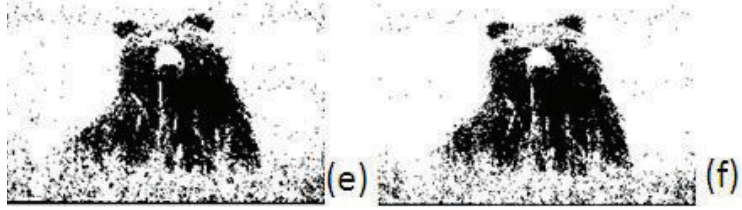

Fig. 9. Clustering results of a real image. (a). Original image, (b). the same image with gaussian noise (var=0.01), (c). FCM results, (d). symFCM results, (e). SFCM results, and (f). S_symFCM results.
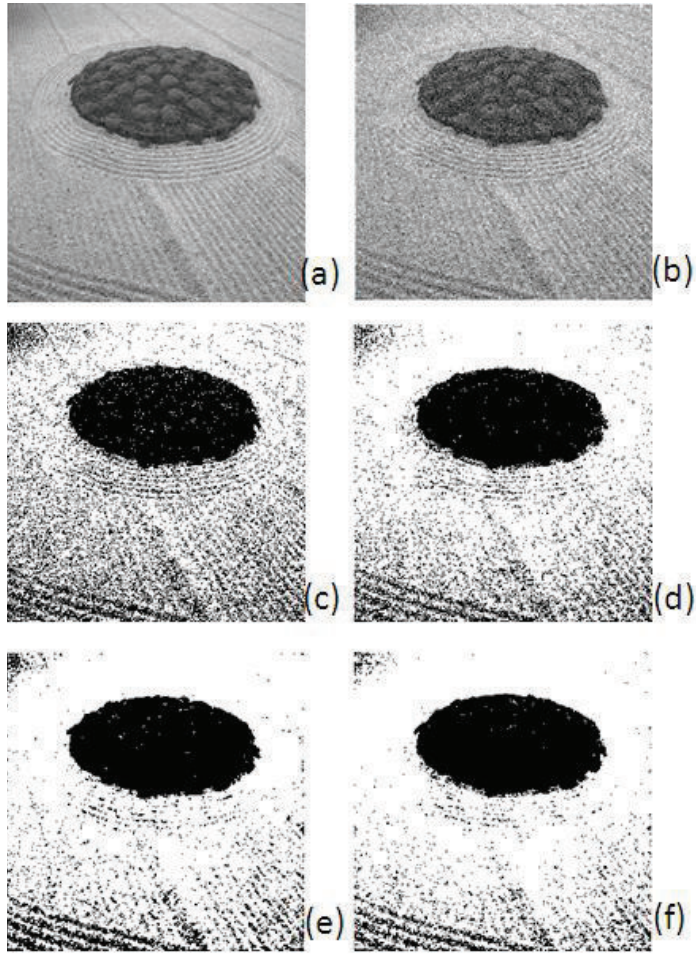

है.

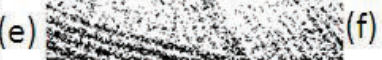

Fig. 10. Clustering results of a real image. (a). Original image, (b). the same image with gaussian noise (var=0.01), (c). FCM results, (d). symFCM results, (e). SFCM results, and (f). S_symFCM results. 


\section{CONCLUSION AND FUTURE WORK}

In this paper, we presented a modified fuzzy c-means algorithm that incorporates symmetry information in order to improve the noise sensitivity in image segmentation. The method was tested on both synthetic and real images. Results showed that the noise sensitivity was considerably reduced in segmentation in comparison with the conventional FCM. We further improved the algorithm with the addition of neighbourhood spatial information. Our results show that symmetry information compliments the spatial continuity information, thus showing improved accuracy and robustness.

Our future work involves a thorough validation of our methods with various symmetrical and non-symmetrical real images, and the extension of our algorithms to deal with multiple local symmetries.

\section{ACKNOWLEDGMENT}

This work was supported by the Australian Research Council (ARC) Discovery Grant DP1097059 and the Australian Postgraduate Award (APA).

\section{REFERENCES}

[1] J. Bezdek, Pattern Recognition with Fuzzy Objective Function Algorithms. Plenum Press, 1981.

[2] M. Ahmed, S. Yamany, N. Mohamed, A. Farag, and T. Moriarty, "A Modified Fuzzy C-Means Algorithm for Biasfield Estimation and Segmentation of MRI Data," IEEE Transactions on Medical Imaging, vol. 21(3), pp. 193-199, 2002.

[3] A. W. C. Liew and H. Yan, "Current methods in the automatic tissue segmentation of 3D magnetic resonance brain images," Current Medical Imaging Reviews, vol. 2, no. 1, pp. 91-103, 2006.

[4] _ - "An adaptive spatial fuzzy clustering algorithm for MR image segmentation," IEEE Transactions on Medical Imaging, vol. 22, no. 9, pp. 1063-1075, 2003.

[5] F. Attneave, "Symmetry, information, and memory for patterns," The American Journal of Psychology, vol. 68, no. 2, pp. 209-222, 1955.

[6] N. Ray, B. Saha, and M. Brown, "Locating brain tumors from MR imagery using symmetry," in Conference Record of The FortyFirst Asilomar Conference on Signals, Systems and Computers, Pacific Grove, CA, 2007, pp. 224-228.

[7] Y. Sun, B. Bhanu, and S. Bhanu, "Automatic symmetryintegrated brain injury detection in MRI sequences," in IEEE Computer Society Conference on Computer Vision and Pattern Recognition Workshops, 2009, pp. 79-86.

[8] Y. Sun and B. Bhanu, "Reflection symmetry-integrated image segmentation," IEEE Transactions on Pattern Analysis and Machine Intelligence, vol. 34(9), pp. 1827-1841, 2012.

[9] G. Loy and J. Eklundh, "Detecting symmetry and symmetric constellations of features," in European Conference on Computer Vision, 2006, pp. 508-521.

[10] D. O'Mara and R. Owens, "Measuring bilateral symmetry in digital images," in IEEE TENKON- Digital Signal Processing Applications, 1996, pp. 151-156.

[11] S. A. Jayasuriya and A. W. C. Liew, "Fractal dimension as a symmetry measure in 3D brain MRI analysis," in IEEE International Conference on Machine Learning and Cybernetics, 2012, pp. 11181123.

[12] _ "Symmetry plane detection in neuroimages based on intensity profile analysis," in IEEE International Symposium on
Information Technology in Medicine and Education (ITME), vol. 2, 2012, pp. 599-603.

[13] A. W. C. Liew, S. Leung, and W. H. Lau, "Fuzzy image clustering incorporating spatial continuity," IEE Proceedings on Vision, Image, and Signal Processing, vol. 147, no. 2, pp. 185-192, 2000.

[14] C. Cocosco, V. Kollokian, R. Kwan, and A. Evans, "Brainweb: Online interface to a $3 \mathrm{~d}$ mri simulated brain database," Neuroimage, vol. 5(4), pp. 425-425, 1997.

[15] X. Ren, C. Fowlkes, and J. Malik, "Figure/ground assignment in natural images," in European Conference on Computer Vision, 2006, pp. 614-627.

[16] D. Martin, C. Fowlkes, D. Tal, and J. Malik, "A database of human segmented natural images and its application to evaluating segmentation algorithms and measuring ecological statistics," in Proc. 8th Int'l Conf. Computer Vision, vol. 2, July 2001, pp. 416-423. 